\title{
Morphological Analysis of Dendritic Spine Development in Primary Cultures of Hippocampal Neurons
}

\author{
Michele Papa, ${ }^{a}$ Marsha C. Bundman, Varda Greenberger, and Menahem Segal \\ Department of Neurobiology, The Weizmann Institute of Science, Rehovot, 76100 Israel
}

We monitored developmental alterations in the morphology of dendritic spines in primary cultures of hippocampal neurons using confocal laser scanning microscopy (CLSM) and the fluorescent marker Dil. Dissociated rat hippocampal neurons were plated on polylysine-coated glass cover slips and grown in culture for 1-4 weeks. Fixed cultures were stained with Dil and visualized with the CLSM. Spine density, spine length, and diameters of spine heads and necks were measured. Some cultures were immunostained for synaptophysin and others prepared for EM analysis. In the 1-3 week cultures, $92-95 \%$ of the neurons contained spiny dendrites. Two subpopulations of spine morphologies were distinguished. At 1 week in culture, "headless" spines constituted $50 \%$ of the spine population and were equal in length to the spines with heads. At 2, 3, and 4 weeks in culture headless spines constituted a progressively smaller fraction of the population and were, on average, shorter than spines with heads. Spines with heads had narrower necks than headless spines. At 3 weeks in culture, spines were associated with synaptophysin-immunoreactive labeling, resembling synaptic terminals. At 4 weeks in culture, only $70 \%$ of the Dilfilled cells had spiny dendrites, and the density of spines decreased. Ultrastructurally, the majority of dendritic spinelike structures at 1 week resembled long filopodia without synaptic contacts. The majority of axospinous synapses were on short "stubby" spines. At 3 weeks in culture, the spines were characteristic of those seen in vivo. They contained no microtubules or polyribosomes, were filled with a characteristic, filamentous material, and formed asymmetric synapses. These studies provide the basis for further analysis of the rules governing the formation, development, and plasticity of dendritic spines under controlled, in vitro conditions.

[Key words: dendritic spines, tissue culture, hippocampus, confocal microscopy, electron microscopy, development, Dil]

Received Sept. 24, 1993; revised April 22, 1994; accepted June 16, 1994.

We gratefully acknowledge the generous gift of monoclonal anti-synaptophysin from BioMakor, Rehovot, Israel. We thank Dr. E. White for consultation on electron micrographs and $\mathrm{O}$. Avni for advice on descriptive statistics. This study was supported in part by an Israel Academy of Sciences and Humanities Basic Research Foundation Grant and a United States-Israel Binational Science Foundation Grant 89/011 to M.S., CNR (Italy) AI93.00151.04 and an Italian-Israeli Bilateral Scientific Relations grant from the Foreign Ministry of Israel to M.P., and a Fogarty International Postdoctoral Fellowship to M.C.B.

Correspondence should be addressed to Dr. Menahem Segal at the above address.

a Present address: Istituto di Anatomia Umana, Facoltà di Medicina e Chirurgia, Seconda Universitả degli Studi di Napoli, 80138, Italy.

Copyright (C) 1995 Society for Neuroscience 0270-6474/95/150001-11\$05.00/0
The dendritic spine constitutes the main locus of excitatory synaptic interaction among central neurons. The great variety of shapes, strategic location, and abundance has focused considerable attention on the dendritic spine as the key site for the potential encoding of activity-dependent, neuronal plasticity. Extensive analyses of hippocampal neurons at the ultrastructural level have indicated that spines undergo marked morphological changes following exposure to various plasticity-producing stimuli, including tetanic stimulation (Lee et al., 1980; Chang and Greenough, 1984; Desmond and Levy, 1988) and kindling (Geinisman et al., 1988). However, accurate electron microscopic analysis has proved to be very tedious and provides a relatively small sample from which to generalize the results (Harris et al., 1989, 1992). The dimension of the spine, being at the limit of optical resolution, has greatly limited the study of spine morphology and function at the light microscope level. However, the recent advent of confocal microscopy in conjunction with high-yield fluorescent marker molecules provides a powerful tool for the high-resolution analysis of changes in spine morphology in relation to neuronal function.

To date, most studies examining alterations in spine morphology associated with neuronal plasticity have utilized in situ or in vitro slice preparations. Tissue cultures afford an ideal environment within which to examine the individual factors that might trigger or regulate alterations in synaptic morphology. Hippocampal dendritic spines have been identified at the ultrastructural level in various tissue culture preparations, including dissociated cells (Bartlett and Banker, 1984), organotypic, rollertube cultures (Zimmer and Gähwiler, 1984), intraocular transplants of embryonic tissue (Sorensen and Zimmer, 1988), and organotypic cultures grown on artificial membranes (Buchs et al., 1993). However, little attempt has been made to study systematically the changes in dendritic spines of cultured neurons. To characterize more fully the plastic changes that spines undergo in culture upon exposure to various plasticity-producing stimuli, we first chose to examine the morphology of spines in the process of development or maturation, processes during which similar changes are expected to take place.

We have developed tissue culture conditions (Segal and Manor, 1992) in which we can grow central neurons for several weeks and monitor changes in their dendrites and dendritic spines via confocal microscopy. Electron microscopy was used to verify that the structures seen at the light level did indeed have the characteristics of spines classically described at the ultrastructural level. The present study provides evidence for the presence of dendritic spines in cullured hippocampal neurons, and analyzes age-dependent changes in these spines. These studies illustrate the potential use of tissue-cultured neurons for the 

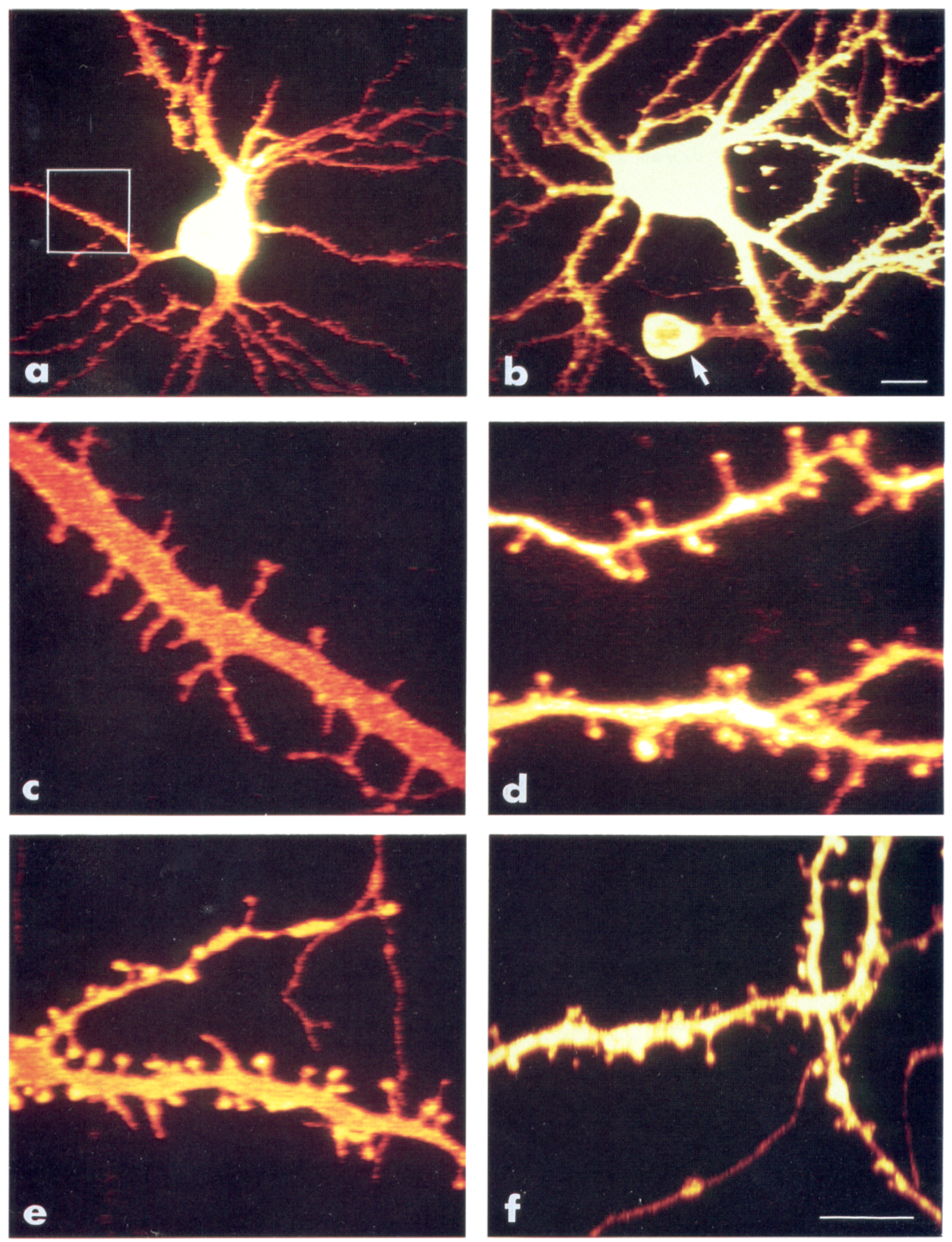
analysis of plasticity-related, morphological changes in central neurons.

\section{Materials and Methods}

Tissue culture. Primary cultures of hippocampus were prepared from 19-d-old Wistar rat embryos, as previously described (Segal and Manor, 1992). The hippocampus was dissected in chilled Leibovitz L15 medium enriched with $0.6 \%$ glucose and in the presence of $15 \mu \mathrm{g} / \mathrm{ml}$ gentamicin. The medium was oxygenated and the dissection carried out at $4^{\circ} \mathrm{C}$. The tissue was mechanically dissociated in small volumes of L15 using a fire-polished Pasteur pipette. Tissue was suspended in plating medium consisting of $5 \%$ fetal calf serum and $5 \%$ heat-inactivated horse serum prepared in Eagle's minimal essential medium enriched with $0.6 \%$ glucose, $2 \mathrm{~mm}$ glutamine, and $15 \mu \mathrm{g} / \mathrm{ml}$ gentamicin. Neurons were plated on poly-L-lysine-coated $(15 \mu \mathrm{g} / \mathrm{ml}) 13 \mathrm{~mm}$ round glass coverslips, (\#1, Chance Propper, Lid., UK), in 24-well culture plates, at a density of 500,000 cells per well. Three to four days after plating, the medium was changed to one containing $10 \%$ dialyzed horse serum. In addition, the first change of medium also contained a mixture of $50 \mu \mathrm{g} / \mathrm{ml}$ uridine, and $20 \mu \mathrm{g} / \mathrm{ml} \mathrm{5}$-fluoro-2 deoxyuridine to block the proliferation of glial cells. Thereafter, the growth medium was changed once a week, if necessary.

Immunohistochemistry. Three-week-old cultures were fixed with $4 \%$ paraformaldehyde in $0.1 \mathrm{~m}$ phosphate buffer, $\mathrm{pH} 7.4$ (PB) for 30 min. Fixed cultures were washed three times $(5 \mathrm{~min}$ each) with $\mathrm{PB}$ and preincubated with $5 \%$ horse serum (Vector Laboratories, Inc., Burlingame, $\mathrm{CA}$ ) in $\mathrm{PB}$ for $1 \mathrm{hr}$ at room temperature. Without rinsing, cultures were incubated with a primary monoclonal antibody against synaptophysin overnight at $4^{\circ} \mathrm{C}(1: 150$ in PB containing $1.5 \%$ horse serum) (BioMakor, Rehovot, Israel). The following morning, the cultures were washed three times ( 5 min each) with PB, and incubated with an FITC-labeled secondary antibody against mouse IgG (1:64; BioMakor, Rehovot, Israel) for $10 \mathrm{~min}$. The cultures were rinsed three times with PB before the cells were labeled with DiI.

Confocal microscopy. At 1-4 weeks following plating, coverslips containing the cultures were taken out of the 24-well plates, washed with $\mathrm{PB}$, and fixed with $4 \%$ paraformaldehyde in PB for $30 \mathrm{~min}$. Fixed cultures, in $\mathrm{PB}$, were placed on the stage of an inverted phase microscope, and individual cells were stained with $1,1^{\prime}$-dioctadecyl-3,3,3', $3^{\prime}$ tetramethylindocarbocyanine perchlorate (DiI; Molecular Probes, Inc., Eugene, OR). The DiI was dissolved in halibut liver oil at saturating concentration, loaded in a 2-5 $\mu \mathrm{m}$ tip micropipette, and applied by pressure ejection onto medium-size $(10-20 \mu \mathrm{m})$ neurons (Hosokawa et al., 1992). The coverslips were placed at $4^{\circ} \mathrm{C}$ in small petri dishes containing PB and 6-24 hr were allowed for transport of the dye before confocal microscopy. A Leica, upright confocal laser scanning microscope (CLSM) equipped with a $488 \mathrm{~nm}$ argon ion laser and a $40 \times, 1.30$ $\mathrm{NA}$, oil-immersion objective was used to visualize individual cells and dendrites. Optical serial sections of $0.3-0.6 \mu \mathrm{m}$ were taken through the cells and reconstructed to yield complete "three-dimensional" images of individual cells in focus.

Those DiI-filled cells considered to have "spiny" dendrites and that were well isolated from other filled cells were retained for quantitative analyses. Three or four pictures were taken from proximal, middle, and distal regions of secondary dendrites (Fig. 1a). For light microscopic analysis, dendritic "spines" were defined as dendritic appendages that protruded for relatively short distances $(<7 \mu \mathrm{m})$ at roughly right angles from the dendrite and had no further branches. Such "spines" were clearly distinct from dendritic branches and bore no resemblance to growth cones (Fig. 1). (Note that no large, multibranched spines of the mossy fiber-CA3, "thorny excrescences" variety were seen in these cultures.) From a selected image, $31 \mu \mathrm{m} \times 31 \mu \mathrm{m}$ (represented on the computer screen by $512 \times 512$ pixels), the length and the total number of spines on that dendritic length were measured using the CLSM Image

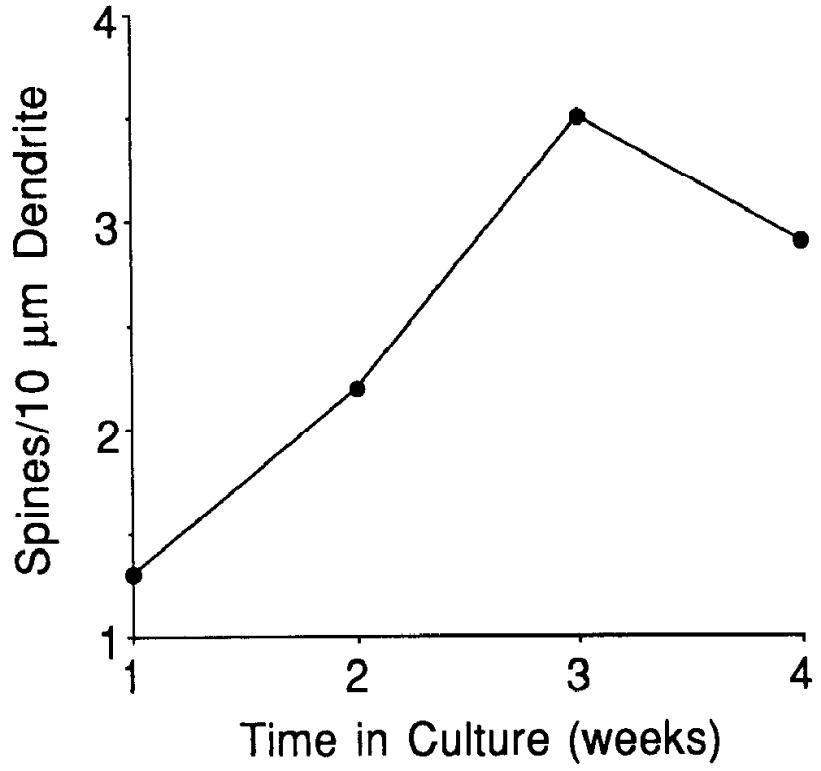

Figure 2. Spine density varies with time in culture. Each point represents the total number of spines divided by the total dendritic length (in micrometers) at each time point, times 10 . There is a steady increase in spine density from 1 to 3 weeks, but spine density decreases from 3 to 4 weeks in culture. See Results for details of spine numbers and dendritic lengths.

Documentation and Analysis software (Leica Lasertechnik GmbH). In addition, the length, the diameter of the head (long axis of obvious heads and the widest part of the "end" of headless spines; Dh), and diameter of the neck (Dn) were measured for each spine. The difference $\mathrm{Dh}$ - Dn was calculated for each individual spine. Due to the optical limits of resolution of the microscope, individual measurements were recorded to the nearest $0.1 \mu \mathrm{m}$, and only the means were expressed in $0.01 \mu \mathrm{m}$. (See note in Discussion regarding optical resolution.) At each age, values were calculated for the number of spines per $10 \mu \mathrm{m}$ of dendritic length, and the means \pm SEM for the length of spines, the diameters of the spine heads and necks, and the difference, $\mathrm{Dh}-\mathrm{Dn}$. Statistical comparisons were madc using one-way analysis of variance (ANOVA) and the Scheffe post hoc $F$ test.

Electron microscopy. Cultures were fixed, first with $3 \%$ glutaraldehyde in culture medium at $37^{\circ} \mathrm{C}$ for $10 \mathrm{~min}$, then with $3 \%$ glutaraldehyde and $1 \%$ osmium tetroxide $\left(\mathrm{OsO}_{4}\right)$, also in culture medium at $37^{\circ} \mathrm{C}$ for $30 \mathrm{~min}$, and finally with $1 \% \mathrm{OsO}_{4}$ in $0.12 \mathrm{M}$ phosphate buffer, $\mathrm{pH} 7.3$, at $37^{\circ} \mathrm{C}, 30 \mathrm{~min}$ (Hasty and Hay, 1978; Rees and Reese, 1981; Deitch and Banker, 1993). The remaining procedures were carried out at room temperature. Cultures were stained en bloc with $2 \%$ aqueous uranyl acetate, dehydrated in ascending ethanols and propylene oxide, and infiltrated overnight with $50 \%$ araldite/Polybed 812 resin in propylene oxide. Glass coverslips containing the cultures were inverted over silicon rubber molds containing fresh resin and allowed to polymerize for $3 \mathrm{~d}$ at $60^{\circ} \mathrm{C}$. Embedded cultures were separated from the coverslips by successive immersion in boiling water and liquid nitrogen (Banker and Goslin, 1991), and selected areas of the blocks were remounted on prehardened resin blocks. Ultrathin silver sections were cut parallel to the culture surface, collected on Formvar-coated single slot copper grids, stained with ethanolic uranyl acetate and lead citrate, and examined with a Philips EM-410 electron microscope.

\section{$\leftarrow$}

Figure 1. Confocal laser scanning images of DiI-filled hippocampal neurons in culture. $a$, Low-power image of a typical, medium-sized, pyramidallike neuron. Analyses of spine density and morphological parameters were taken from secondary dendrites (box). $b$, Low-power image of a large, multipolar cell. A small granule cell (arrow) was also filled by the same drop of DiI. $c-f$, Examples of dendrites from $1-4$ week cultures, respectively, demonstrating the differences in the density and morphology of dendritic spines. The dendritic protrusions seen at 1 week $(c)$ are very long without prominent spine heads. At 2 and 3 weeks $(d, e)$, the spines are shorter and have narrower necks, giving the appearance of larger spine heads. The density of spines increases from 1 to 3 weeks. At 4 weeks $(f)$, spine heads are not as prominent and spine density has decreased. Scale bars: $a$ and $b, 10.0 \mu \mathrm{m} ; c-f, 5.0 \mu \mathrm{m}$. 

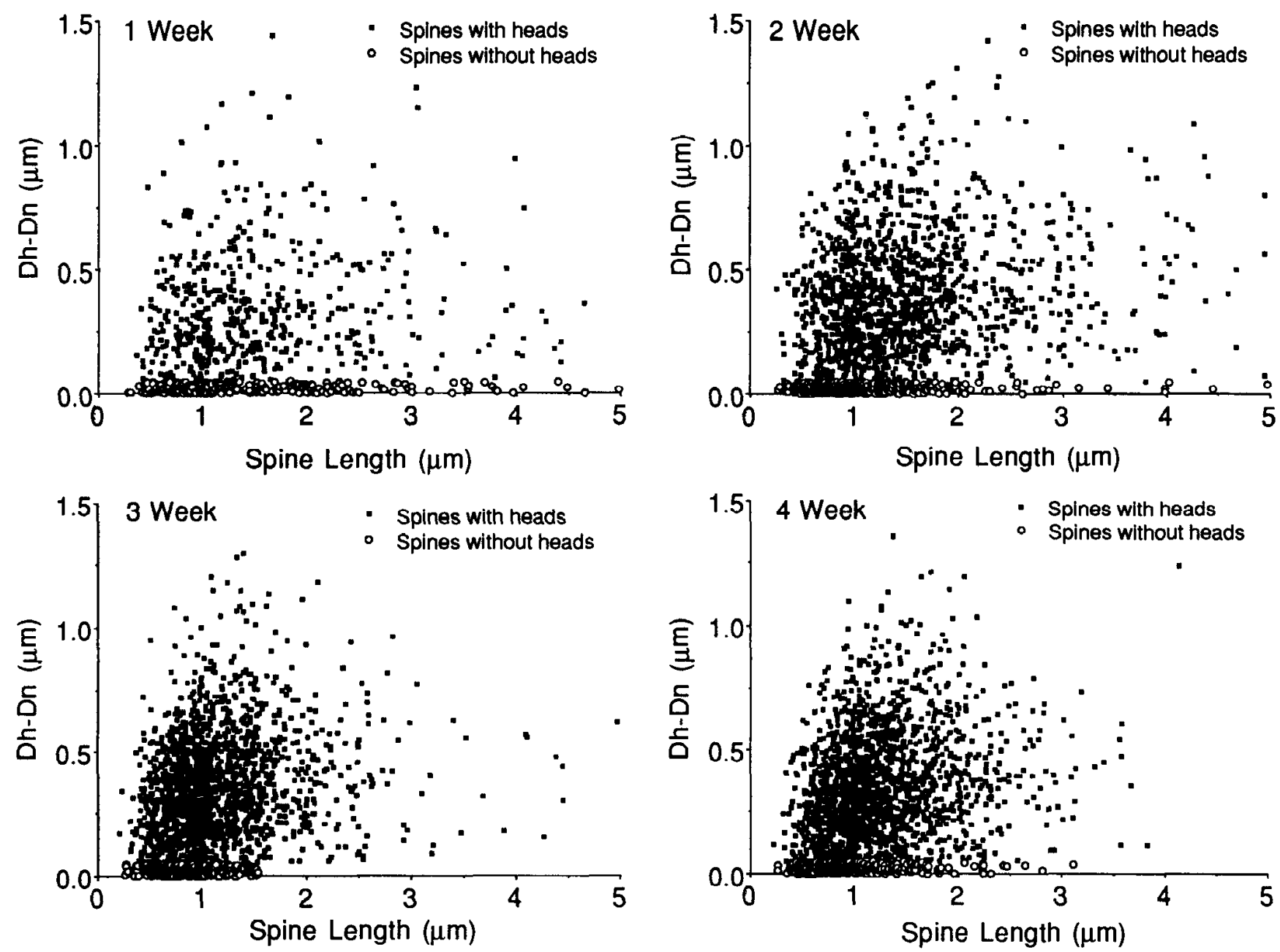

Figure 3. Scatter plots of spine length versus the difference Dh - Dn (representing the prominence of spine heads). The number of points at each time is given by $N$ in Table 1 . Two distinct subpopulations of spine-like structures are distinguishable. Spines without heads $(O)$, where $\mathrm{Dh}-\mathrm{Dn}$ is equal to zero, constitute one-half of the spines measured at 1 week but represent a far smaller fraction of the population at later times in culture. Spines with discernible heads $(\square)$, where $\mathrm{Dh} \cdots$ Dn is greater than zero, become increasingly homogeneous with time in culture.

\section{Results}

\section{Confocal microscopy}

Rat hippocampal cultures consisted primarily of pyramidal-like, medium-sized neurons (10-20 $\mu \mathrm{m}$ in diameter) with 2-4 long (over $100 \mu \mathrm{m}$ ) dendrites extending from the somata. Some cells were large and multipolar, with many dendrites lacking a distinct polarity. Some small round neurons $(\sim 10 \mu \mathrm{m}$ in diameter) with a single primary dendrite resembled dentate gyrus gr ule cells. Most of the labeled cells belonged to the first category (Fig. $1 a, b)$.

The following measurements were made from a single series of cultures grown under identical conditions for $1-4$ weeks. Preliminary experiments were conducted with 3-4 series of cultures and yielded similar values for spine dimensions and density. One of these experiments involved labeling of live cells with DiI. Spine dimensions appeared similar in the live and fixed cultures. However, since the live cells tended to deteriorate, and expressed large morphological changes subsequent to this deterioration, we chose to conduct the present study with fixed cells. Another preliminary experiment involved a comparison of spine dimensions measured by two independent observers, which yielded similar results.
A total dendritic length of $45,583 \mu \mathrm{m}$ was analyzed and a total of 11,063 spines were counted in cultures from $1-4$ weeks of age. The totals were composed of 509 separate dendritic segments from the 1 week cultures and 529, 390, and 292 dendritic segments from the 2,3 , and 4 week cultures, respectively. The dendritic segments were from 30-40 neurons in each case. The analysis of spine morphology was obtained from 1018 spines in the 1 week cultures and 2433,2505 , and 2618 spines in the 2, 3, and 4 week cultures, respectively.

As shown in Figure 2, there was a large, steady increase in the density of dendritic spines between 1 week and 3 weeks in culture resulting in 2.7 times as many spines per $10 \mu \mathrm{m}$ of dendritic length. However, at 4 weeks in culture the density of spines declined. Spine densities increased from 1.3 spines $/ 10$ $\mu \mathrm{m}$ at 1 week to $2.2,3.5$, and 2.9 spines $/ 10 \mu \mathrm{m}$ of dendritic length at 2, 3, and 4 weeks, respectively. The percentage of DiIfilled neurons that had "aspiny" dendrites was fairly constant from 1 to 3 weeks ( $8-5 \%$ ); however, in the 4 week culture, fully $30 \%$ of the cells had "aspiny" dendrites. Included in the latter population were some neurons that had only 20-30 spines scattered over the entire dendritic arbor. The density of spines on dendrites did not appear to vary as a function of the density of live cells remaining in the culture dish, as dendrites with a high 

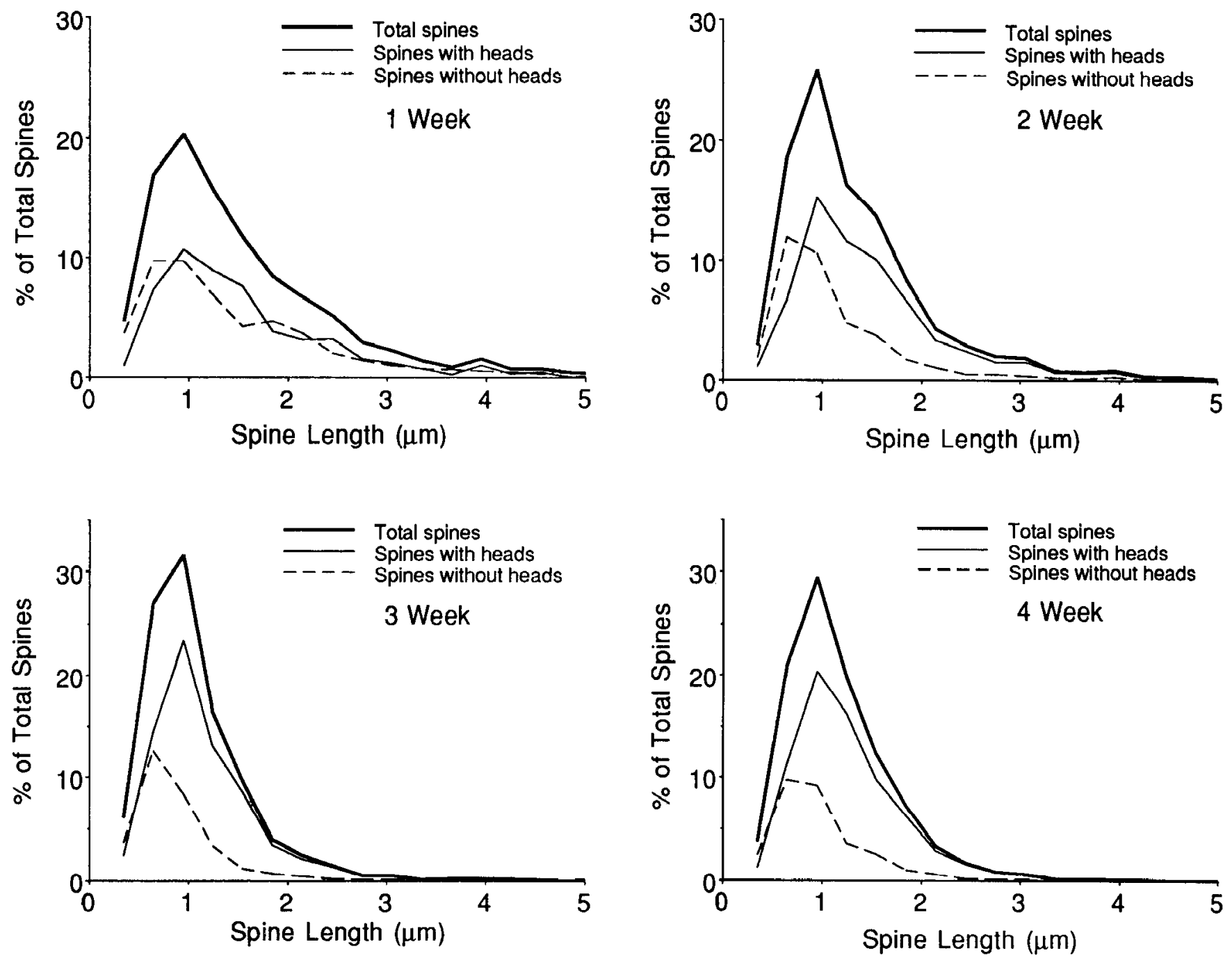

Figure 4. Frequency distributions of spine length data. Histograms are plotted for total spines (thick solid lines) and for the subpopulations of spines with heads (thin solid lines) and spines without heads (dashed lines). Bin widths are $0.3 \mu \mathrm{m}$ and the number of spines in each category is expressed as the percentage of the total spines at the various time points. Note the dramatic decrease in the frequency of long, headless spines at 2 weeks in culture, compared to that at 1 week, and the increased homogeneity of the spines with heads at 3 weeks in culture.

density of spines were sometimes found on cells that were fairly isolated in the culture.

The analysis of spine shape revealed significant differences in morphology at different times in culture (Fig. $1 c-f$ ). In addition, careful inspection of spine lengths, and diameters of spine heads $(\mathrm{Dh})$ and necks $(\mathrm{Dn})$ revealed the presence of two distinct populations of spine morphologies, those with heads (Dh - Dn > 0 ) and those without heads $(\mathrm{Dh}-\mathrm{Dn}=0)$. This is best demonstrated in a two-dimensional scatter plot of length versus the value $\mathrm{Dh}$ - Dn (Fig. 3), where the headless spines are congregated along the $\mathrm{x}$-axis. In the 1 week cultures, headless spines constituted $50 \%$ of the spine population and were equally distributed in length with the spines possessing heads (Fig. 4, Table 1). At 2 weeks there was a dramatic decrease in the population of "long" headless spines; by 3 and 4 weeks in culture headless spines constituted only $30 \%$ of the total spine population, and these were almost all less than $1.0 \mu \mathrm{m}$ and most were not longer than $1.5 \mu \mathrm{m}$ in length (Fig. 3). With increased time in culture, dendritic spines with heads constituted increasingly larger fractions of the spine population. The average length of dendritic spines with heads at 3 weeks was only $75 \%$ of those at 1 week in culture.

In addition to the changes in average spine lengths across the various ages, there were also differences in the heterogeneity of spine lengths within the age groups. In the younger cultures, the spine population was highly variable and was increasingly more homogeneous with increased time in culture. This was evident in the shape of the frequency histograms (Fig. 4) and in the decreased SEMs shown in Table 1. This can also be seen in the scatter plots in Figure 3, where the points are widely dispersed at 1 week and are increasingly concentrated with increasing length of time in culture. Most of the variability existed in spines among different neurons and not in spines within the same neuron. This was most apparent in the 2 week cultures, where there were sometimes cells that looked like those in 1 week cultures as well as cells that looked like those in 3 week cultures in proximity to one another. While there did not appear to be any obvious corrclation between the sizes or shapes of neurons and the length of their dendritic spines, there did appear to be a correlation between density and spine length. That is, in the 


\begin{tabular}{llrlllll}
\hline Table 1. & \multicolumn{7}{l}{} \\
$\begin{array}{llrllll}\text { Time in } \\
\text { culture }\end{array}$ & Heads & $N$ & $\%$ & Length $(\mu \mathrm{m})$ & Dh $(\mu \mathrm{m})$ & Dn $(\mu \mathrm{m})$ & Dh - Dn $(\mu \mathrm{m})$ \\
\hline 1 Week & + & 515 & 51 & $1.54 \pm 0.04$ & $0.65 \pm 0.01$ & $0.29 \pm 0.01$ & $0.35 \pm 0.01$ \\
& - & 503 & 49 & $1.46 \pm 0.04$ & $0.42 \pm 0.01 \dagger$ & $0.45 \pm 0.01 \dagger$ & \\
2 Weeks & + & 1518 & 62 & $1.50 \pm 0.02$ & $0.73 \pm 0.01^{*}$ & $0.33 \pm 0.01$ & $0.40 \pm 0.01^{*}$ \\
& - & 915 & 38 & $1.15 \pm 0.03^{*} \dagger$ & $0.49 \pm 0.01^{*} \dagger$ & $0.50 \pm 0.01^{*} \dagger$ & \\
3 Weeks & + & 1744 & 70 & $1.15 \pm 0.01^{*}$ & $0.62 \pm 0.01$ & $0.25 \pm 0.004^{*}$ & $0.37 \pm 0.01$ \\
& - & 761 & 30 & $0.87 \pm 0.02^{*} \dagger$ & $0.42 \pm 0.01^{\dagger}$ & $0.42 \pm 0.01 \dagger$ & \\
4 Weeks & + & 1855 & 71 & $1.25 \pm 0.01^{*}$ & $0.59 \pm 0.01^{*}$ & $0.23 \pm 0.003^{*}$ & $0.36 \pm 0.01$ \\
& - & 763 & 29 & $0.96 \pm 0.02^{*} \dagger$ & $0.34 \pm 0.01^{*} \dagger$ & $0.34 \pm 0.01^{*} \dagger$ &
\end{tabular}

Values represent the mean ( \pm SEM) lengths, and diameters of heads (Dh) and necks (Dn), expressed in micrometers for the number of spines given by $N$. Dh $-\mathrm{Dn}$ is the mean of all the individual differences.

* Significantly different from the comparable measurement at 1 week (ANOVA, Scheffe post $F$ test, $95 \%$ confidence).

$\dagger$ Significantly different from the value "with heads" of the same age.

2 week cultures, the cells with long spines had low spine densities (similar to cells at 1 week) and the cells with shorter, 3 weeklike spines had a high density of spines. At 3 and 4 weeks the lengths of spines were far more homogeneous.

There were also differences in the dimensions of the heads (or head "areas") and necks of the headless spines and those with prominent heads. The average diameter of the head region of spines in which Dh - Dn was greater than zero was approximately $50 \%$ larger than in spines in which Dh was equal to Dn. Concomitantly, the average diameter of the necks of spines with heads was nearly $40 \%$ less than the necks of the headless spines.
Thus, the prominence of spine heads appears to be a factor of both the enlargement of the head regions and a narrowing of spine necks. There did not appear to be any consistent change in these characteristics contingent on the time in culture.

\section{Immunocytochemistry}

Synaptophysin is a synaptic vesicle protein (Jahn et al., 1985) and, as such, labels presynaptic terminals. In the 3-4 week cultures, synaptophysin-immunoreactive (SFI) boutons were clustered around somata of large pyramidal-shaped neurons. SFI boutons were also seen along dendrites and juxtaposed to den-
Figure 5. Confocal laser scanning image of secondary dendrites from DiIfilled hippocampal neurons in cultures FITC-immunostained for synaptophysin. $a$, Image taken of the fluorescence from both the DiI- and the FITC-tagged anti-synaptophysin. $b$, Image taken following the photobleaching of the more labile FITC, thus representing only the DiI staining. Comparison of the two images shows that many synaptophysin-immunoreactive (SFI) terminal boutons can be seen closely associated with dendritic spines (arrowheads). SFI staining that is not associated with the DiI-labeled cell is probably associated with other, unlabeled cells. $c$, Two similar images from an aspiny dendrite. Comparison of the two images shows that SFI is concentrated on the dendritic shaft. Scale bar applies to $a-c$.
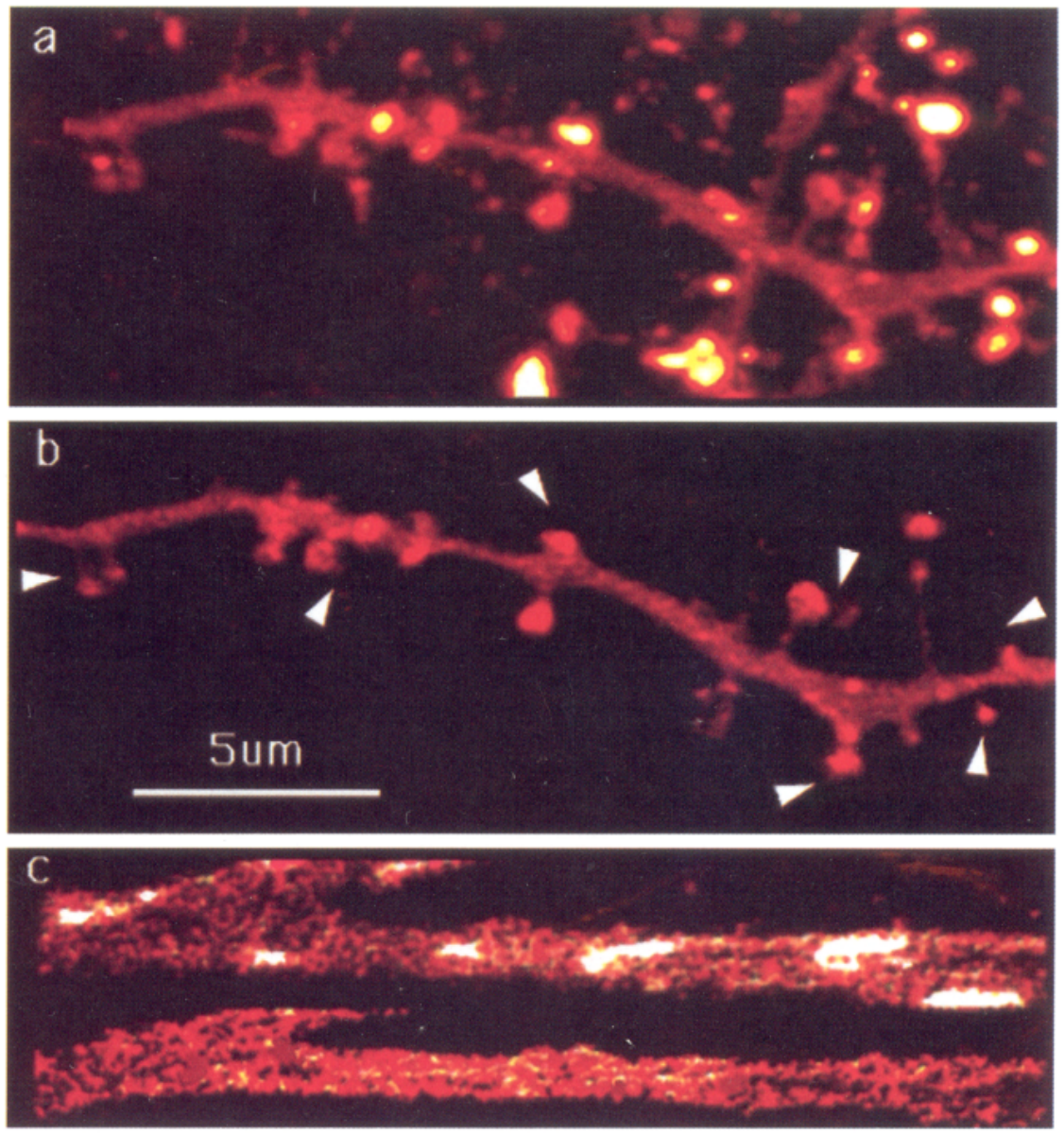

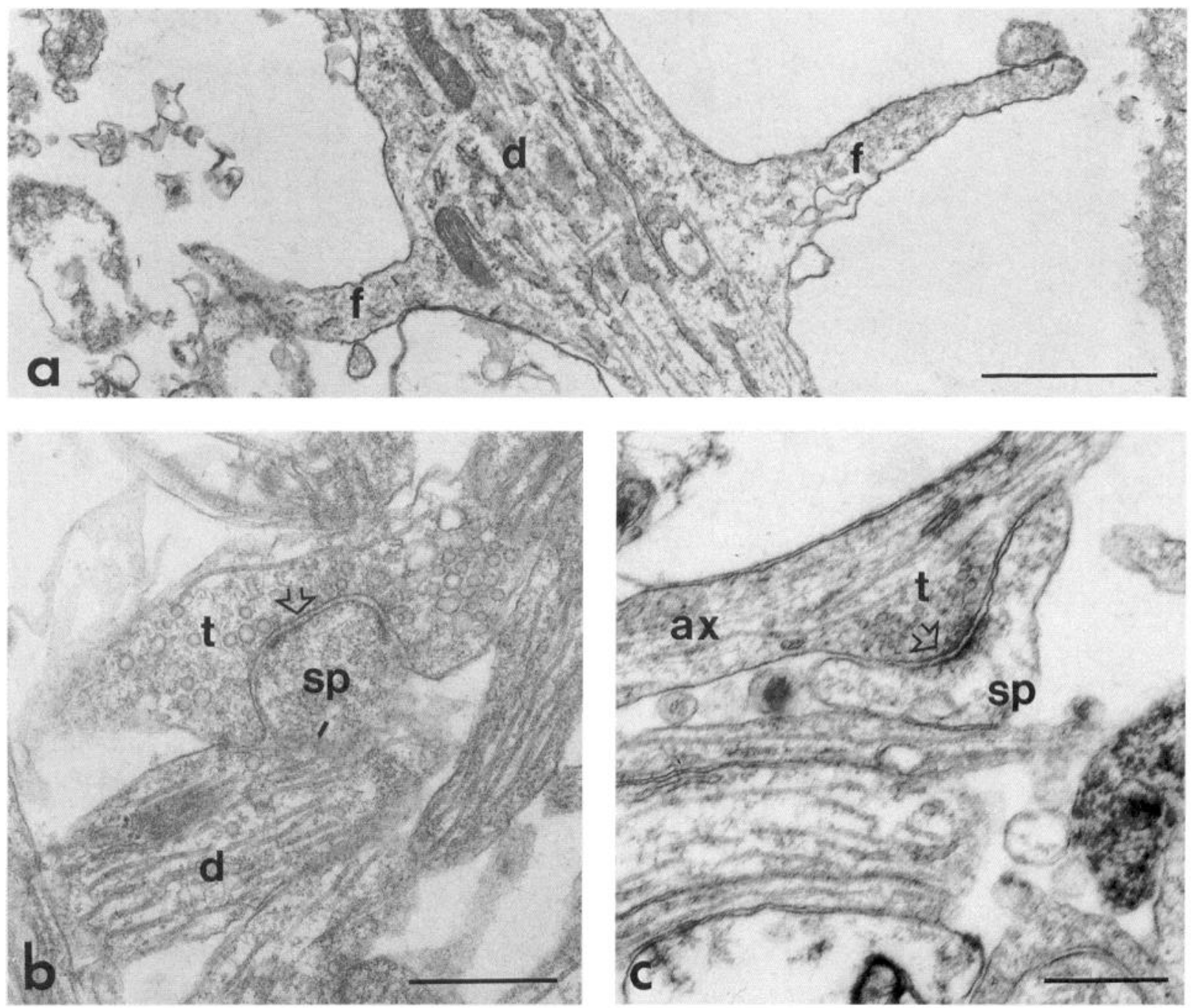

Figure 6. Electron micrographs of 1-2-week-old primary hippocampal neuronal cultures. $a$, A length of dendrite with two long, noninnervated filopodia $(f)$ from a 1-week-old culture. $b$, A synapse (open arrow) on a short "stubby" dendritic spine from a 1-week-old culture. $c$, A mushroom-shaped spine head $(s p)$ from a 2-week-old culture forms an asymmetric synapse (open arrow) with an en passant axonal varicosity. ax, axon; $d$, dendrite; $f$, filopodia; $s p$, dendritic spine; $t$, presynaptic terminal or varicosity. Scale bars: $a, 1.0 \mu \mathrm{m} ; b$ and $c, 0.5 \mu \mathrm{m}$.

dritic spines (Fig. 5a,b). While a large number of dendritic spines were associated with SFI boutons, it was clear that some spines were not. At this level of analysis, it is not possible to determine whether these SFI-negative spines did or did not form synapses. In several aspiny neurons stained with DiI, the SFI reaction product was localized adjacent to dendritic shafts (Fig. $5 c$ ), whereas in the spiny neurons, most of the SFI boutons were associated with the spines.

\section{Electron microscopy}

The most conspicuous feature of the electron microscopic preparations from 1-week-old hippocampal cultures was the many long, thin, pedunculate appendages seen protruding from dendrites. Many of these filopodia-like profiles were greater than 2 $\mu \mathrm{m}$ long and did not terminate in enlarged heads, typical of spines. However, these profiles contained the same flocculent or filamentous material that is classically described in dendritic spines (Peters et al., 1976). They were easily distinguished from dendritic branches in that microtubules coursing through the dendrite scrupulously avoided entering the spine-like structures
(Fig. 6a). In addition, no mitochondria or polyribosomes were found in these structures. Many had clusters of membranous sacs at the base of the profile. Although asymmetric synapses were seen on short "stubby" spines (Fig. $6 b$ ) and on dendritic shafts, synapses were never seen on the long filopodia. Some filopodia were seen in proximity to profiles loosely filled with vesicles but examination of adjacent sections did not reveal the presence of synaptic membrane specializations.

At 2 weeks in culture, few of the long, noninnervated filopodia were seen and dendritic spines with distinct spine heads and asymmetric synapses were clearly evident (Fig. 6c). Many short "stubby" spines were seen, as were spines with narrow necks and simple, enlarged spine heads. Both formed asymmetric synapses with terminal boutons or axonal varicosities (Fig. 6c).

By 3-4 weeks in culture, dendritic spines were far more prevalent than at either 1 or 2 weeks. The spines at this age ranged in shape from small, "stubby," or "sessile" spines (Fig. $7 d$ ) to ones with very complex-shaped spine heads (Fig. 7e). However, most typically had thin necks and mushroom-shaped heads that formed synapses with terminal boutons or with en passant ax- 

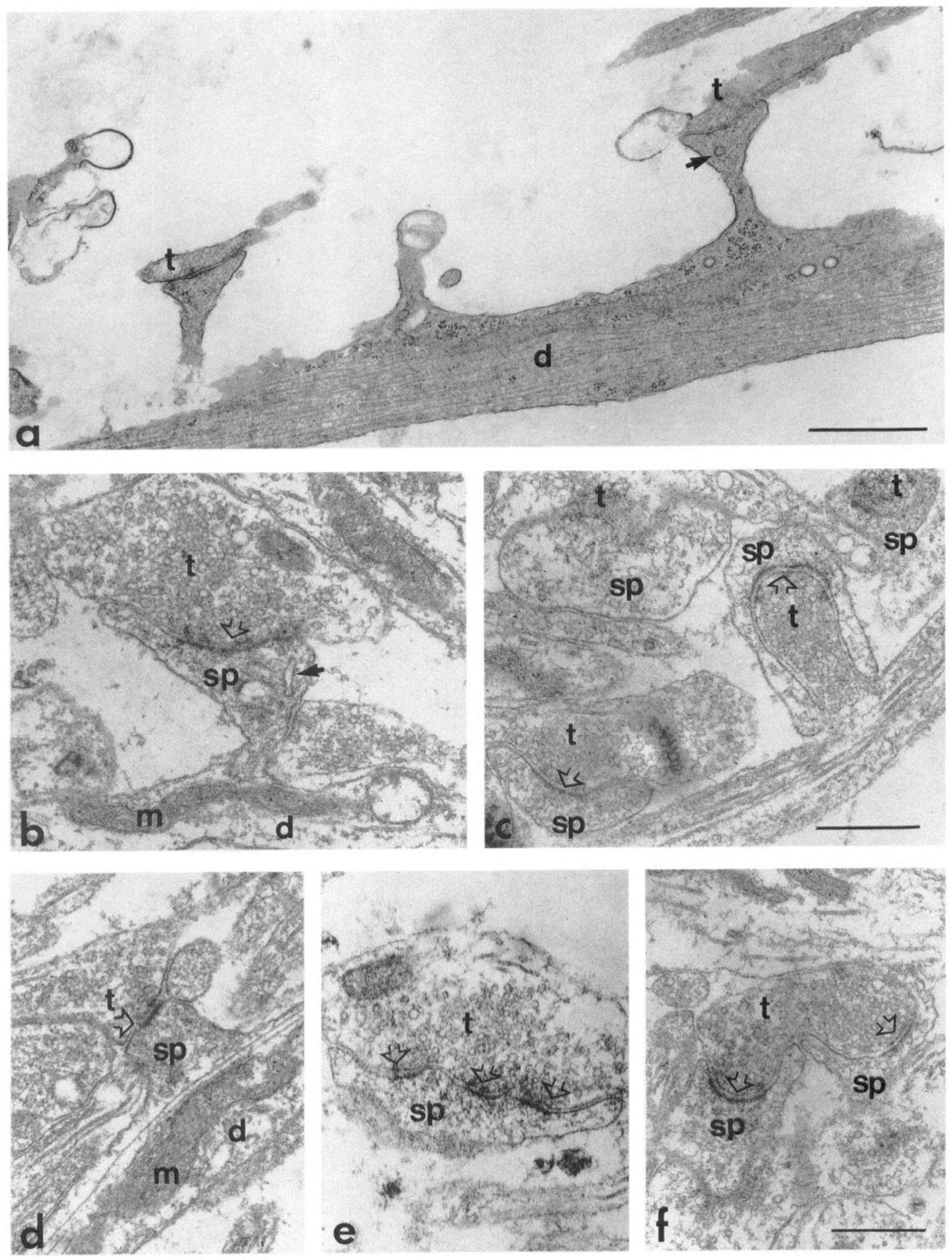
onal varicosities (Fig. 7a,b). This particular class of spines was seen in continuity with the parent dendrite in only a small fraction of the cases; however, isolated profiles of mushroomshaped spine heads were plentiful (Fig. $7 c$ ) and easily identified by the presence of a dense flocculent or filamentous material and the conspicuous lack of microtubules, mitochondria, and polyribosomes (Peters et al., 1976). The spine heads and sometimes the spine necks also contained dilated cisternae of smooth endoplasmic reticulum. While an extensive, quantitative study was not undertaken to measure spines at the ultrastructural level, measurements of spines in available micrographs revealed dimensions within the same ranges as those obtained with the CLSM.

In all cases in which specialized synaptic membranes were clearly discernible, the synapses on spines were of the asymmetric type. That is, the postsynaptic membrane thickening was denser than that of the presynaptic side and the presynaptic elements contained round, electron-lucent, synaptic vesicles. Many instances were found of large spine heads that received synapses from more than one presynaptic element or multiple, perforated synapses from the same presynaptic terminal (Fig. $7 e$ ). In the latter case, the presynaptic terminals were generally larger than others and contained large numbers of densely packed synaptic vesicles. There were also several occurrences of single presynaptic terminals that formed synapses with more than one spine (Fig. $7 f$ ).

\section{Discussion}

Changes in the morphology of dendritic spines are believed to represent one mechanism by which recent synaptic activity leaves its imprint on neurons. While electron microscopy provides the most detailed descriptions of such changes, light level analyses afford a global view that is lacking at the ultrastructural level. We used confocal laser scanning microscopy, in combination with DiI labeling, to show that, in the course of development in tissue culture, dendritic spines undergo changes in density and morphology that are easily measured at the light microscope level. In primary cultures of hippocampal neurons, there was an increase in the density of dendritic spines from 1 week to 4 weeks in culture and a concomitant decrease in the length of spines and in the percentage of headless spines. Prominent spine heads appear to be a result of both the enlargement of the tips of spines and the narrowing of spine necks. Moreover, the population of spines at 3-4 weeks was far more homogeneous than those at earlier ages.

The dimensions of dendritic spines in culture, measured at the light level, are well within the range of those measured ultrastructurally from in situ preparations (Harris et al., 1992). An obvious concern presented here is the measurement of objects (i.e., spine neck diameters) near the resolution limits of the fluorescent microscope. The specifications of our particular optics yield a theoretical Abbe resolution limit of $0.23 \mu \mathrm{m}$. However, it has been shown that with the use of confocal microscopy and a small field of view, this theoretical limit can be increased by a factor of two (McCutchen, 1967; Wilson and Sheppard, 1984), thus producing an effective lateral resolution in the range of $0.1 \mu \mathrm{m}$. While the mean spine neck diameters varied from $0.23 \mu \mathrm{m}$ to $0.50 \mu \mathrm{m}$, many individual necks measured $0.1 \mu \mathrm{m}$. To determine the reliability of these measurements, we measured fluorescent-labeled latex beads (Molecular Probes, Inc., Eugene, OR) with diameters of $0.3 \mu \mathrm{m}$ and $0.1 \mu \mathrm{m}$. Both of these could be easily seen in the CLSM and were reproducibly measured and distinguished from one another. Thus, we feel confident in our ability to measure structures of this size with the CLSM.

We observed a $170 \%$ increase in the density of spines from 1 to 3 weeks in culture. However, even at 3 weeks there was only one-third the density of spines as that reported for in situ Golgi preparations (Woolley et al., 1990) or from EM reconstructions of PND 15 rat hippocampus (Harris et al., 1992). Several possible explanations can be offered for this discrepancy. First, we did not include the "hidden" spines that protrude in back of or in front of the dendrites. However, assuming dendritic diameters approximately equal to the average spine length (see Fig. 1), and considering that in monolayer cultures the dendrites grow on the surface of the culture dish, there were probably very few spines that actually projected from behind the dendrite; methodological considerations probably accounted for a loss of only about $10 \%$ of the spines (Braitenberg and Schüz, 1991).

The second possible explanation assumes that the normal rate of spine proliferation is disrupted by the process of cell dissociation and the cells never fully recover from this insult. Thus, while cells in culture for 3 weeks are chronologically similar to those at about PND 20 in situ, many growth- and maturationrelated processes may have been interrupted or suspended during the process of culturing. It is also evident that the cultures are grown in nutrient conditions different from those in vivo. Also, neurons growing in culture do not receive the same degree of afferent input as those in situ. It is apparent, when looking at electron micrographs, that there is much more empty "space" in the cultures compared to in situ. In situ, the neurons themselves are much more densely packed than in culture; thus, there is greater chance of contact between cells. In addition, many sources of afferent activity (e.g., the massive afferent input from the entorhinal cortex or the brainstem) are completely missing in the culture conditions. Thus, a much reduced source of afferent input may have led to the reduction in postsynaptic spines.

Finally, it is possible that neurons are programmed to have an intrinsic, predetermined number of spines. In situ, hippocampal neurons and their dendrites observe very strict bound-

Figure 7. Electron micrographs of 3-4-week-old primary hippocampal neuronal cultures, showing dendrites and dendritic spines. The dendrites are largely filled with microtubules while the spines contain no microtubules or polyribosomes but are filled with a fluffy, flocculent material also characteristic of dendritic spines in situ. $a$, A length of dendrite with three dendritic spines. Notice that polyribosomes are especially concentrated at the base of spines. The two mushroom-shaped spine heads sit on top of thin necks and receive synapses from en passant axon terminals $(t)$. There is a small cisterna of endoplasmic reticulum in the head of the spine to the far right (arrow). $b$, Higher-power micrograph of a dendritic spine ( $(s p)$ with a mushroom-shaped spine head. Dilated cisternae of endoplasmic reticulum can be seen (solid arrow), as well as a large asymmetric synapse (open arrow). c, A field of "neuropil" from a 3 week culture, with four isolated spine heads ( $s p$ ), demonstrating the high density of spines seen at this age. $d$, An example of a short, "stubby" dendritic spine that receives an asymmetric synapse (open arrow) from an axon terminal. $e$, An example of a large, complex spine head with a perforated synapse (open arrows) from a large presynaptic terminal. $f$, An example of a presynaptic terminal that makes synapses (open arrow) with two different spines. $d$, dendrite; $m$, mitochondria; $s p$, spine; $t$, presynaptic terminal. Scale bars $=$ $a, 1.0 \mu \mathrm{m} ; b-f, 0.5 \mu \mathrm{m}$. 
aries, resulting in a very ordered, highly laminated structure (Blackstad, 1956). In culture, such boundaries are lacking and dendritic arbors extend over far greater distances and in all directions. Thus, it is possible that cells in culture have spine numbers similar to those in situ but the longer dendrites result in lower numbers of spines/dendritic length.

The decrease in spine density and in the number of cells with spiny dendrites at 4 weeks may be indicative of an aging process in culture. Other signs of aging in these cells include a marked increase in autofluorescence akin to that associated with increased lipofuscin deposits in aging cells (Brizzee and Ordy, 1981) and the fact that most of the cells die before 2 months in culture. It is assumed that the cultures are composed largely of the two groups of pyramidal cells from regions CA3 and CA1, but the hippocampus as a whole is made up of a heterogeneous population of cells that have not been individually identified in these cultures. While the CA3 and CA1 pyramidal cells have roughly the same density of dendritic spines (Gould et al., 1990a,b), other populations of hippocampal cells may have more or less spiny dendrites. Therefore, we cannot rule out the possibility that both the decrease in the number of cells with spiny dendrites and the decrease in the density of spines per dendrite represents a preferred survival of aspiny or less spiny neurons. However, the presence of a subpopulation of cells with an extremely low density of spines that were seen only at 4 weeks indicates that some cells begin losing spines between 3 and 4 weeks in culture. Similar decreases in spine density have been associated with aging in vivo (Feldman and Dowd, 1974; Scheibel et al., 1975; Geinisman and Bondareff, 1976). In addition, the low density of spines at this time in culture is probably not related entirely to a loss of cells and the subsequent loss of possible inputs to the spines. As mentioned above, some very spiny cells were sometimes found quite isolated from other cells in the culture dish; hence, it is not obvious that spine density should be correlated with cell density.

A major question, yet unresolved, concerns the emergence of new spines. That is, are spines formed first and make synapses later, or do existing synapses grow to form spines? At 1 week, a large percentage of spine-like appendages seen at the ultrastructural level were noninnervated, filopodia-like processes. Although the filopodia seen in culture possessed the same internal ultrastructure as spines (i.e., presence of a flocculent, filamentous material and membranous sacs and a lack of microtubules, polyribosomes, or mitochondria), they did not form synapses, and thus it is difficult to classify them formally as spines. At 2 weeks far fewer of the long "filopodia-like" structures were present, and at 3 weeks they were seldom seen, an observation confirmed by quantification at the light microscopic lcvel (Fig. 4). Concomitantly, shorter spines with discernible heads increased in number. One might postulate, therefore, that the filopodia seen in young cultures were precursors of more mature spines and that, upon formation of synapses, the filopodia shrink and form classic spines with typical spine heads. Time-lapse recordings of hippocampal neurons in culture have demonstrated that once stable contacts are made between axonal growth cones and preexisting, target cell filopodia, the filopodia do shrink (Cooper and Smith, 1992).

It is also possible that innervated spines with thin necks and enlarged heads develop from synapses that form on the dendritic shaft. In the young cultures, asymmetric synapses were found predominantly on dendritic shafts and on short stubby spines. Also, from 2 to 3 weeks in culture, the period of greatest increase in spine density, almost no filopodia were found. If spines start first as long filopodia and, upon synapse formation, they contract to form classic spines, then one would expect to find many such filopodia during the period of greatest spine proliferation. Thus, filopodia are not prerequisites for spine formation. That spines develop directly from dendritic shaft synapses was suggested earlier by developmental studies in visual cortex (Juraska and Fifkova, 1979; Miller and Peters, 1981) and was further supported by developmental evidence gathered from electron microscopic, 3-D reconstructions in rat hippocampus (Harris et al., 1989) and visual cortex (Schweizer, 1990). In neither of the latter studies were spines found without synapses. Evidence also exists for the interconversion of synapses directly on dendritic shafts with those on stubby spines, following long-tcrm potentiation (LTP) in rat hippocampal slices (Chang and Greenough, 1984), and for the seizure-induced elaboration of somatic spines at the sites of preexisting synapses on dentate granule cells (Bundman and Gall, 1994). The presence of noninnervated filopodia has also been described in situ but their function is unclear. Perhaps in very young cultures or early in normal brain development filopodia serve to attract approaching axonal contacts (Cooper and Smith, 1992), and in more established cultures, or upon neuronal maturation, spines arise directly from dendritic synapses. Experiments in which repeated observations could be made of the same living cells would greatly help to resolve this question.

Upon ultrastructural analysis, the spines seen at the light level in 3 and 4 week cultures bear all the characteristics of hippocampal dendritic spines seen in situ. Dendritic spines on pyramidal cells are classically associated with asymmetric synapses (Gottlieb and Cowan, 1972), as were the spines seen in culture. Several of the complex types of spine synapses that have been previously correlated with a variety of plasticity-inducing paradigms in vivo or in hippocampal slices are also present in the cultures. These include perforated synapses, associated with kindling, aging, and memory (de Toledo-Morrell et al., 1984; Morrell and de Toledo-Morrell, 1986; Geinisman et al., 1988); concave synapses on mushroom-shaped spine heads, associatcd with LTP (Desmond and Levy, 1988); and spines with convergent synapses, associated with synaptogenesis (Matthews et al., 1976). Such occurrences suggest the possibility of testing the mechanisms by which such plastic changes occur.

Along with other recent studies, the present results indicate that dendritic spines undergo marked morphological changes. Thus, the dendritic spine is a dynamic structure highly responsive to changes in ambient conditions. Our use of a confocal microscope, together with a highly fluorescing membrane probe, promises to be a most useful tool in the detection of such morphological changes in spincs. These studies will lead to a better understanding of the morphological changes in central neurons that underlie neuronal plasticity.

\section{References}

Banker G, Goslin K (1991) Characterizing and studying neuronal cultures. In: Culturing nerve cells (Banker G, Goslin K, eds), pp 75109. Cambridge, MA: MIT Prcss.

Bartlett WP, Banker GA (1984) An electron microscopic study of the development of axons and dendrites by hippocampal neurons in culture. II. Synaptic relationships. J Neurosci 4:1954-1965.

Blackstad TW (1956) Commissural connections of the hippocampal region of the rat, with special reference to their mode of termination. J Comp Neurol 105:417-538.

Braitenberg V, Schüz A (1991) Dendritic spines. In: Anatomy of the cortex: statistics and geometry, pp 113-118. Bcrlin: Springer. 
Brizzee KR, Ordy JM (1981) Cellular features, regional accumulation, and prospects for modification of age pigments in mammals. In: Age pigments (RS Sohal, eds), pp 101-154. Amsterdam: Elsevier/NorthHolland.

Buchs P-A, Stoppini L, Muller D (1993) Structural modifications associated with synaptic development in area CAl of rat hippocampal organotypic cultures. Dev Brain Res 71:81-91.

Bundman MC, Gall C (1994) Ultrastructural plasticity of the dentate gyrus granule cells following recurrent limbic seizures. II. Alteration of somatic synapses. Hippocampus, in press.

Chang F-LF, Greenough WT (1984) Transient and enduring morphological correlates of synaptic activity and efficacy change in the rat hippocampal slice. Brain Res 309:34-46.

Cooper MW, Smith SJ (1992) $\Lambda$ real-time analysis of growth conetarget cell interactions during the formation of stable contacts between hippocampal neurons in culture. J Neurobiol 23:814-828.

Deitch JS, Banker GA (1993) An electron microscopic analysis of hippocampal neurons developing in culture: early stages in the emergence of polarity. J Neurosci 13:4301-4315.

Desmond N, Levy $W$ (1988) Anatomy of associative long-term synaptic modification. In: Long-term potentiation: from biophysics to behavior (Landfield PW, Deadwyler SA, eds), pp 265-305. New York: Liss.

de Toledo-Morrell L, Morrell F, Fleming S (1984) Age dependent deficits in spatial memory are related to impaired hippocampal kindling. Behav Neurosci 98:902-907.

Feldman ML, Dowd C (1974) Aging in rat visual cortex: light microscopic observations on layer V pyramidal apical dendrites. Anat Record 178:355.

Geinisman Y, Bondareff W (1976) Decrease in the number of synapses in the senescent brain: a quantitative electron microscopic analysis of the dentate gyrus molecular layer in the rat. Mech Ageing Dev 5:11-23.

Geinisman Y, Morrell F, de Toledo-Morrell L (1988) Remodeling of synaptic architecture during hippocampal "kindling." Proc Natl Acad Sci USA 85:3260-3264.

Gottlieb DI, Cowan WM (1972) On the distribution of axonal terminals containing spheroidal and flattened synaptic vesicles in the hippocampus and dentate gyrus of the rat and cat. Z Zellforsch 129: $413-429$.

Gould E, Allan MD, McEwen BS (1990a) Dendritic spine density of adult hippocampal pyramidal cells is sensitive to thyroid hormone. Brain Res 525:327-329.

Gould E, Woolley CS, Frankfurt M, McEwen BS (1990b) Gonadal steroids regulate dendritic spine density in hippocampal pyramidal cells in adulthood. J Neurosci 10:1286-1291.

Harris KM, Jensen FE, Tsao BH (1989) Ultrastructure, development, and plasticity of dendritic spine synapses in area CAl of the rat hippocampus: extending our vision with serial electron microscopy and three-dimensional analyses. In: The hippocampus: new vistas (Chan-Palay V, Köhler C, eds), pp 33-52. New York: Liss.

Harris KM, Jensen FE, Tsao BH (1992) Three-dimensional structure of dendritic spines and synapses in rat hippocampus (CAl) at post- natal day 15 and adult ages: implications for the maturation of synaptic physiology and long-term potentiation. J Neurosci 12:26852705 .

Hasty DL, Hay ED (1978) Freeze-fracture studies of the developing cell surface. II. Particle-free membrane blisters on glutaraldehydefixed corneal fibroblasts are artifacts. J Cell Biol 78:756-768.

Hosokawa T, Bliss TVP, Fine A (1992) Persistence of individual dendritic spines in living brain slices. Neuroreport 3:477-480.

Jahn R, Schiebler W, Ouimet C, Greengard P (1985) A 38,000-dalton membrane protein (p38) present in synaptic vesicles. Proc Natl Acad Sci USA 82:4137-4141.

Juraska JM, Fifkova E (1979) A Golgi study of the early postnatal development of the visual cortex of the hooded rat. J Comp Neurol 183:247-256.

Lee KS, Schottler F, Oliver M, Lynch G (1980) Brief bursts of highfrequency stimulation produce two types of structural change in the rat hippocampus. J Neurophysiol 44:247-258.

Matthews DA, Cotman C, Lynch G (1976) An electron microscopic study of lesion-induced synaptogenesis in the dentate gyrus of the adult rat. II. Reappearance of morphologically normal synaptic contacts. Brain Res 115:23-41.

McCutchen CW (1967) Superresolution in microscopy and the Abbe resolution limit. J Opt Soc Am 57:1190-1192.

Miller M, Peters A (1981) Maturation of rat visual cortex. II. A combined Golgi-electron microscope study of pyramidal neurons. J Comp Neurol 203:555-573.

Morrell F, de Toledo-Morrell L (1986) Kindling as a model of neuronal plasticity. In: Kindling 3 (JA Wada, ed), pp 17-33. New York: Raven.

Peters A, Palay SL, Webster HF (1976) The fine structure of the nervous system: the neurons and supporting cells. Philadelphia: Saunders.

Rees RP, Reese TS (1981) New structural features of freeze-substituted ncuritic growth concs. Ncuroscience 6:247-254.

Scheibel ME, Lindsay RD, Tomiyasu U, Scheibel AB (1975) Progressive dendritic changes in aging human cortex. Exp Neurol 47: 392-403.

Schweizer M (1990) Zur entwicklung der dendritischen Dornen im Cortex der Ratte. Eine elektronenmikroskopishe Untersuchung. Tübingen: University of Tübingen.

Segal M, Manor D (1992) Confocal microscopic imaging of $\left[\mathrm{Ca}^{2+}\right]_{i}$ in cultured rat hippocampal neurons following exposure to $N$-methylD-aspartate. J Physiol (Lond) 448:655-676.

Sorensen T, Zimmer J (1988) Ultrastructural organization of normal and transplanted rat fascia dentata. I. A qualitative analysis of intracerebral and intraocular grafts. J Comp Neurol 265:15-42.

Wilson T, Sheppard C (1984) Theory and practice of scanning optical microscopy. New York: Academic.

Woolley CS, Gould E, Frankfurt M, McEwen BS (1990) Naturally occurring fluctuation in dendritic spine density on adult hippocampal pyramidal neurons. J Neurosci 10:4035-4039.

Zimmer J, Gähwiler BH (1984) Cellular and connective organization of slice cultures of the rat hippocampus and fascia dentata. J Comp Neurol 228:432-446. 